Original Research

\title{
Neurofilament light as a biomarker of axonal degeneration in patients with mild cognitive impairment and Alzheimer's disease
}

\author{
Yi Chen ${ }^{1}$, Joseph Therriault ${ }^{2}$, Jing Luo ${ }^{3}$, Maowen Ba ${ }^{4}$, Hua Zhang ${ }^{3, * *}$, Alzheimer's Disease Neuroimaging Initiative ${ }^{\S}$ \\ ${ }^{1}$ Department of Radiology, Chongaing Red Cross Hospital (Jiangbei District People's Hospital), 400020 Chongqing, China \\ ${ }^{2}$ The McGill University Research Centre for Studies in Aging, McGill University, Montreal, QC H2L, Canada \\ ${ }^{3}$ Department of Neurology, The First Affiliated Hospital of Chongqing Medical University, 400016 Chongging, China \\ ${ }^{4}$ Department of Neurology, Yantai Yuhuangding Hospital Affiliated to Qingdao Medical University, 264000 Shandong, China \\ "Correspondence: zhanghuapro@hospital.cqmu.edu.cn (Hua Zhang) \\ $\S$ Alzheimer's Disease Neuroimaging Initiative, Data used in preparation of this article were obtained from the Alzheimer's Disease \\ Neuroimaging Initiative (ADNI) database (adni.loni.usc.edu). As such, the investigators within the ADNI contributed to the design and \\ implementation of ADNI and/or provided data but did not participate in analysis or writing of this report. A complete listing of ADNI \\ investigators can be found at: http://adni.loni.usc.edu/wpcontent/uploads/how_to_apply/ADNI_Acknowledgement_List.pdf
}

\section{DOI:10.31083/j.jin2004088}

This is an open access article under the CC BY 4.0 license (https://creativecommons.org/licenses/by/4.0/).

Submitted: 17 June 2021 Revised: 12 October 2021 Accepted: 26 October 2021 Published: 30 December 2021

Cerebrospinal fluid neurofilament light and plasma neurofilament light concentrations are elevated in patients with mild cognitive impairment and Alzheimer's disease. We investigated the clinical relevance of increased neurofilament light concentrations in mild cognitive impairment and Alzheimer's disease patients. In this study, 244 subjects were divided into cognitively normal control $(n=67)$, stable mild cognitive impairment $(n=52)$, progressive mild cognitive impairment $(n=68)$, and Alzheimer's disease $(n=57)$. Linear regression examined the relationships between neurofilament light levels in cerebrospinal fluid or plasma and the diagnostic group. The relationships between neurofilament light and other biomarkers were assessed by Spearman correlation. Linear mixed-effects models were used to test cerebrospinal fluid and plasma neurofilament light as predictors of Alzheimer's disease characteristics, including cognition, cortical glucose metabolism, and brain structure. Cerebrospinal fluid and plasma neurofilament light levels were significantly elevated in Alzheimer's disease. Still, the correlations between neurofilament light and other cerebrospinal fluid biomarkers within the diagnostic groups were often not statistically significant. In addition, the diagnostic accuracy of cerebrospinal fluid and plasma neurofilament light for progressive mild cognitive impairment and Alzheimer's disease was almost the same as that of cerebrospinal fluid total tau (T-tau). It is phosphorylated tau (Ptau) and high cerebrospinal fluid. Neurofilament light predicted conversion from mild cognitive impairment to Alzheimer's disease. A high neurofilament light is related to poor cognition, low cerebral metabolism, hippocampal atrophy, and ventricular enlargement caused by Alzheimer's disease. Our work further identifies cerebrospinal fluid neurofilament light and plasma neurofilament light as biomarkers of axonal degeneration in patients with mild cognitive impairment and Alzheimer's disease.

\section{Keywords}

Neurofilament light; Alzheimer's disease; Mild cognitive impairment; Cerebrospinal fluid

\section{Introduction}

Alzheimer's disease (AD) is the main cause of dementia, which is characterized by extracellular accumulation of aggregated $\beta$-amyloid $(\mathrm{A} \beta)$, intracellular aggregation of hyperphosphorylated tau, and synaptic dysfunction [1, 2]. The failure of several disease-modifying therapies AD highlights the need to further explore diverse disease mechanisms and biomarkers alterations that characterize AD [3-7]. Cerebrospinal fluid (CSF) $\mathrm{A} \beta 42$ and tau have been used to diagnose $\mathrm{AD}$ and monitor disease progression [8]. In the past decade, other biomarkers have been confirmed to further characterize the pathophysiological process of $\mathrm{AD}$ [9].

Neurofilaments are the structural components of axons, which can be measured in CSF $[10,11]$. In CSF, neurofilament proteins (cytoskeletal protein of neurons), including neurofilament light chain (NFL), were associated with axonal degeneration in various diseases, including AD [12]. Several reports have suggested that CSF NFL are elevated early in the $\mathrm{AD}$ process and are associated with longitudinal neurodegeneration and cognitive decline [13]. Interestingly, recent studies have demonstrated that concentrations of NFL derived from plasma were elevated in patients with $\mathrm{AD}$ and related to CSF $\mathrm{A} \beta$ and tau and positron emission tomography (PET) [14-16]. 
However, the diagnostic value of CSF NFL or plasma NFL (NFL in plasma) for mild cognitive impairment (MCI) or AD is unclear [14, 17]. Similarly, it is not clear whether the combination of CSF NFL and plasma NFL can improve the diagnostic accuracy of MCI and AD, or whether CSF or plasma NFL forecasts progression from cognitively normal (CN) control to MCI or as from MCI to AD. The purpose of this study is, therefore, to examine the hypotheses that the combination of CSF and plasma NFL improve the diagnostic accuracy for MCI and AD and whether CSF and plasma NFL predict progression from $\mathrm{MCI}$ to $\mathrm{AD}$ or from $\mathrm{CN}$ to $\mathrm{MCI}$ or AD in participants enrolled in the Alzheimer's Disease Neuroimaging Initiative (ADNI) study [18]. We also assess whether CSF or plasma NFL is related to cognitive dysfunction, brain structure, and cerebral metabolism.

\section{Materials and methods}

\subsection{Subjects description}

Data for this work came from the ADNI database. The subjects participating in this study were between 55 and 90 years old. All subjects completed lumbar puncture, Clinical Dementia Rating scale (CDR), Mini-Mental State Examination (MMSE), and Alzheimer's Disease Assessment Scale cognitive subscale 11 (ADAS-cog 11) assessments. Furthermore, all participants had magnetic resonance imaging (MRI) and 18F-Fluorodeoxyglucose-positron emission tomography (FDG-PET). Based on clinical and behavioral measurements provided by $\mathrm{ADNI}$, these participants were divided into $\mathrm{CN}$ $(\mathrm{n}=67)$, stable MCI (sMCI, $\mathrm{n}=52$ ), progressive MCI (pMCI, $\mathrm{n}=68)$, and AD dementia $(\mathrm{n}=57)$.

\subsection{Classification criteria}

The CN criteria include MMSE score 26-30 and CDR of $0[19,20]$. The MCI criteria consisted of subjective memory complaints, MMSE score 24-30, CDR of 0.5, and retained activities of daily living [21]. In addition to the NINCDS/ADRDA criteria, AD individuals had MMSE scores of 20-26 and CDR scores of 0.5 or 1.0 [22]. We referred to MCI subjects who did not progress to AD during a follow-up period of at least 2 years as $\mathrm{MCI}$ and $\mathrm{MCI}$ participants who developed to $\mathrm{AD}$ at any time during the follow-up period as pMCI [23].

\subsection{CSF and plasma analyses}

As described previously [24], Multiple xMAP Luminex platforms and Innogenetics INNO-BIA AlzBio3 immunoassay reagents were used to measure the levels of CSF A $\beta 42$, total tau (T-tau), and phosphorylated tau (P-tau). A water buffer solution containing a combination of three biomarkers was used to draw a calibration curve for each biomarker. The concentration range of the three biomarkers was as follows: (1) synthetic $\mathrm{A} \beta 1-42$ peptide was 27 to $1574 \mathrm{pg} / \mathrm{mL}$, (2) recombinant tau was 56 to $1948 \mathrm{pg} / \mathrm{mL}$, and (3) the synthetic peptide of tau phosphorylated at position 181 of threonine was 8 to $230 \mathrm{pg} / \mathrm{mL}$. CSF NFL was tested using a commercial ELISA for NFL (NF-light ${ }^{\circledR}$ ELISA, Uman Diagnostics,
Umeå, Sweden) [13, 25]. The lower limit of quantification for this CSF NFL was $50 \mathrm{ng} / \mathrm{L}$. Values were given as $\mathrm{pg} / \mathrm{mL}$. Plasma NFL concentrations were tested by Single-Molecule array (Simoa) technology using a homebrew kit. All samples except one (due to technical reasons) were measured in duplicate. ${ }^{1}$ MMSE and ADAS-Cog-11 scores evaluated global cognitive function ${ }^{2}$. We selected the baseline scores of MMSE and ADAS-Cog 11. We also selected the baseline hippocampal and ventricular volumes ${ }^{3}$. The neuroimaging methods of ADNI have been described previously [26].

\subsection{FDG-PET}

The baseline data of ADNI PET images were collected and processed according to the descriptions by Landau et al. [27]. In short, we applied the mean PET SUVRs in anterior cingulate, lateral and medial frontal lobes, lateral temporal lobes, lateral parietal, and posterior cingulate [23]. $\left({ }^{18} \mathrm{~F}\right)$ FDG-PET values were normalized using the pons as a reference region.

\subsection{Statistical methods}

Cohort demographics were examined using chi-square analysis and analysis of covariance (ANOVA). Multivariate linear regression was used to measure the relationship between CSF NFL or plasma NFL and clinical diagnostic group, adjusted for age and gender.

The correlation between CSF NFL or plasma NFL and other core biomarkers was examined using Spearman correlation. The overall diagnostic accuracy (area under the receiver operator characteristics curve, AUC) of each biomarker was obtained by Receiver operating curve (ROC) analyses. The difference of AUC between two pairs of different biomarkers was measured using bootstrapping.

To examine the relationship between CSF NFL or plasma NFL and AD dementia, Cox proportional hazard regression analysis (adjusted age and gender) calculated hazard ratios (HR) with 95\% CIs. According to the median of each biomarker, CSF NFL and plasma NFL were divided into two groups in Cox proportional hazard regression analyses.

Baseline values of MMSE, ADAS-cog 11, hippocampal and ventricular volumes, and FDG-PET SUVRs were obtained from the intercepts of linear mixed-effects models. The intercepts were subsequently used in linear regression models, and the CSF and plasma NFL values were used as the predictor of clinical diagnostic groups adjusted for age and gender. MMSE and ADAS-cog-11 analyses were additionally corrected for education. Hippocampal and ventricular volume analyses were additionally corrected for intracranial volume. All statistics were conducted using SPSS version 20

\footnotetext{
1 All CSF data in our work were from the ADNI files "UPENNBIOMK5-8.csv", "BLENNOWCSFNFL.csv", and "ADNI_BLENNOWPLASMANFL_10_03_18.csv".

2 The data of this work were from ADNI files "MMSE.csv" and "ADAS_ADNI1. csv".

3 Neurodegeneration, hippocampal and ventricular volumes were obtained from the ADNI files "FOXLABBSI_08_04_17.csv" and "UCSDVOL.csv".
} 
Table 1. Demographics of subjects at baseline.

\begin{tabular}{lcccc}
\hline Characteristics & $\mathrm{CN}(\mathrm{n}=67)$ & $\mathrm{sMCI}(\mathrm{n}=52)$ & $\mathrm{pMCI}(\mathrm{n}=68)$ & $\mathrm{AD}(\mathrm{n}=57)$ \\
\hline Age (years) & $75.2(0.6)$ & $73.4(1.1)$ & $72.9(0.9)$ & $74.3(1.1)$ \\
Gender (F\%) & $30(44.8 \%)^{b}$ & $13(25.0 \%)^{a, d}$ & $28(41.2 \%)$ & $28(49.1 \%)^{b}$ \\
Education (years) & $15.9(0.3)$ & $15.6(0.4)$ & $15.9(0.4)$ & $15.1(0.4)$ \\
APOE $\varepsilon 4(\mathrm{n} \%)$ & $17(25.4 \%)^{b, c, d}$ & $26(50.0 \%)^{a, d}$ & $44(64.7 \%)^{a}$ & $40(70.2 \%)^{a, b}$
\end{tabular}

Post hoc analysis provided significant differences between groups: ${ }^{a}$ from $\mathrm{CN} ;{ }^{b}$ from sMCI;

${ }^{c}$ from $\mathrm{pMCI} ;{ }^{d}$ from AD.
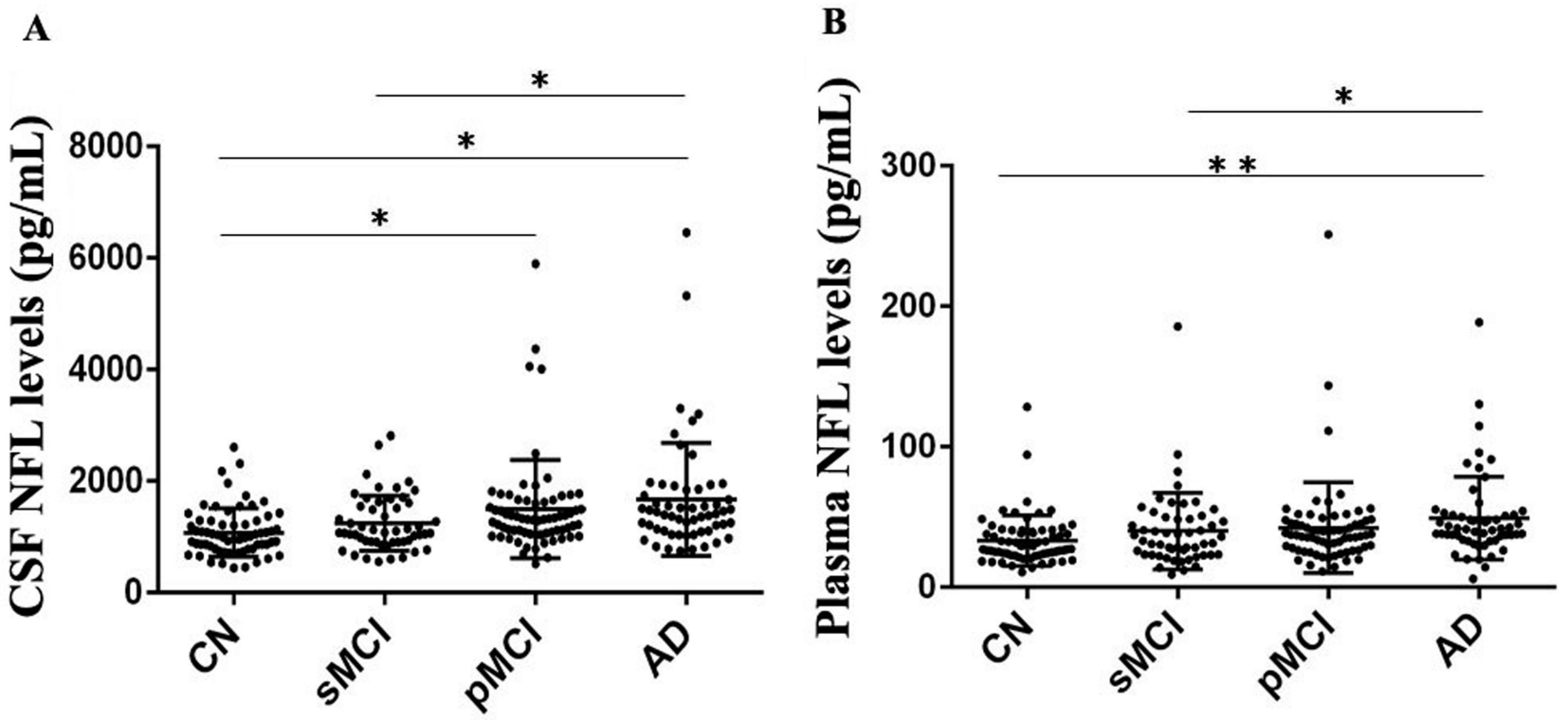

Fig. 1. The levels of CSF and plasma NFL in different groups. The levels of CSF NFL (A) and plasma NFL (B) in different groups. Differences among groups were detected by multiple-variable linear regression. ${ }^{*} p<0.05 ;{ }^{* *} p<0.01$.

(SPSS Inc., Chicago, IL, USA). The statistical significance of all analyses was defined as $p<0.05$.

\section{Results \\ 3.1 Baseline characteristics}

The biomarker characteristics and demographics of all subjects are shown in Table 1 . There were no differences in age or education among clinical groups. There were significantly fewer female individuals in the sMCI group compared to $\mathrm{CN}$ and $\mathrm{AD}$ groups. Apolipoprotein $\mathrm{E}(A P O E) \epsilon 4$ carriership was more common in $\mathrm{AD}$ than $\mathrm{CN}$ and sMCI and more common in $\mathrm{pMCI}$ than $\mathrm{CN}$.

\subsection{The levels of NFL in different groups}

CSF NFL concentrations in pMCI and AD were higher than that in $\mathrm{CN}(p<0.05)$, and CSF NFL levels in AD were higher than that in sMCI $(p<0.05)$, but there was no significant difference between pMCI and $\mathrm{AD}$, and between sMCI and pMCI (Fig. 1A). There were significant differences in plasma NFL levels between $\mathrm{CN}$ and $\mathrm{AD}$, and between sMCI and $\mathrm{AD}$. There was also no significant difference between pMCI and AD, or between sMCI and pMCI (Fig. 1B).

\subsection{Associations between NFL and other biomarkers}

CSF NFL related to plasma NFL in all diagnostic groups (Fig. 2A and Table 2). There was a negative relationship between CSF NFL and A $\beta 42$ in CN subjects (Table 2). However, there were no significant correlations between CSF NFL and $\mathrm{A} \beta 42$ in sMCI, pMCI, and AD individuals (Fig. 2B and Table 2). CSF T-tau was strongly related to CSF NFL in $\mathrm{CN}$ and $\mathrm{AD}$, but not in sMCI and pMCI (Fig. 2C and Table 2). P-tau was only strongly related to CSF NFL in CN, but not in sMCI, pMCI, and AD (Fig. 2D and Table 2). Plasma NFL did not relate to $\mathrm{A} \beta 42$ in all diagnostic groups (Fig. $3 \mathrm{~A}$ and $\mathrm{Ta}-$ ble 2). CSF $\mathrm{T}$-tau was strongly correlated with plasma NFL in pMCI but not in CN, sMCI, and AD (Fig. 3B and Table 2). CSF P-tau did not correlate with plasma NFL in all diagnostic groups (Fig. 3C and Table 2).

\subsection{Diagnostic accuracy of NFL and other CSF biomarkers}

ROC analyses were used to detect CSF biomarkers associated with sMCI, pMCI, and AD clinical diagnoses. Except for the combination of CSF NFL and plasma NFL, other biomarkers did not have significantly different diagnostic accuracy for sMCI (Fig. 4A and Table 3). All biomarkers had significant accuracy in diagnosing pMCI (Fig. 4B and Table 3) 
A
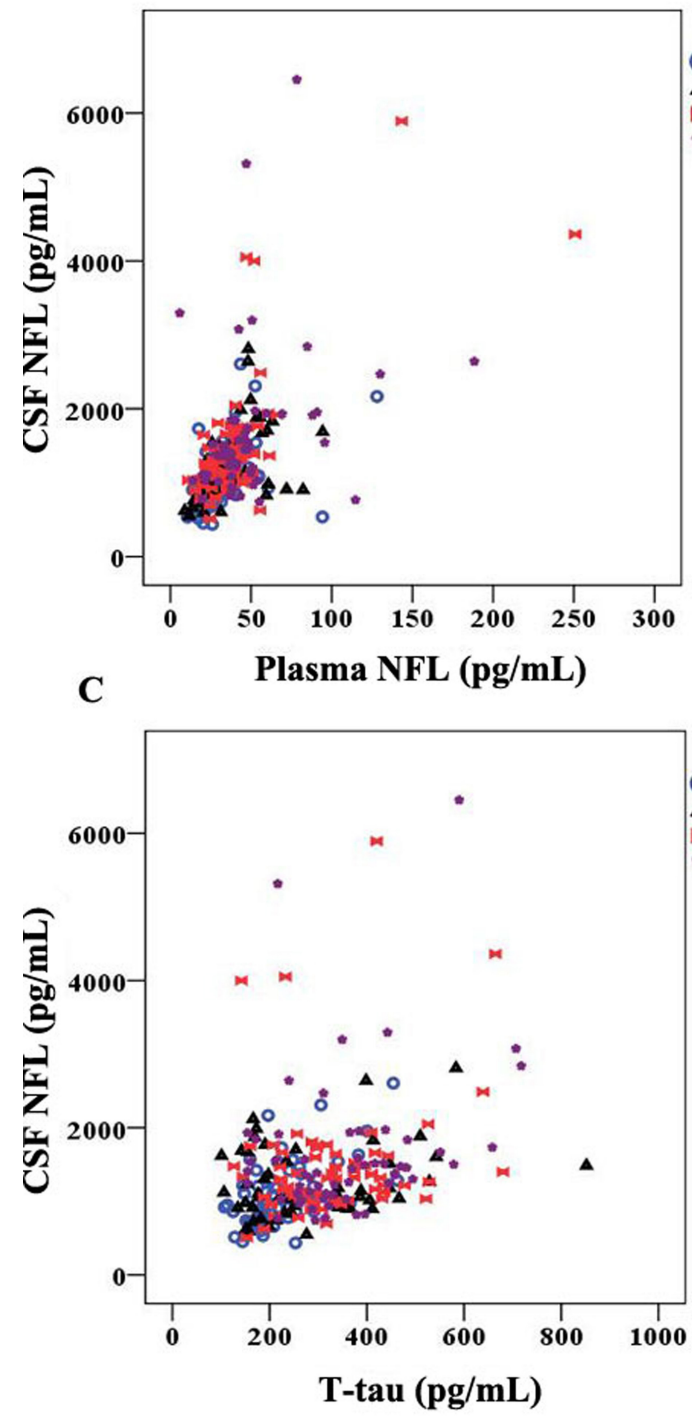

B
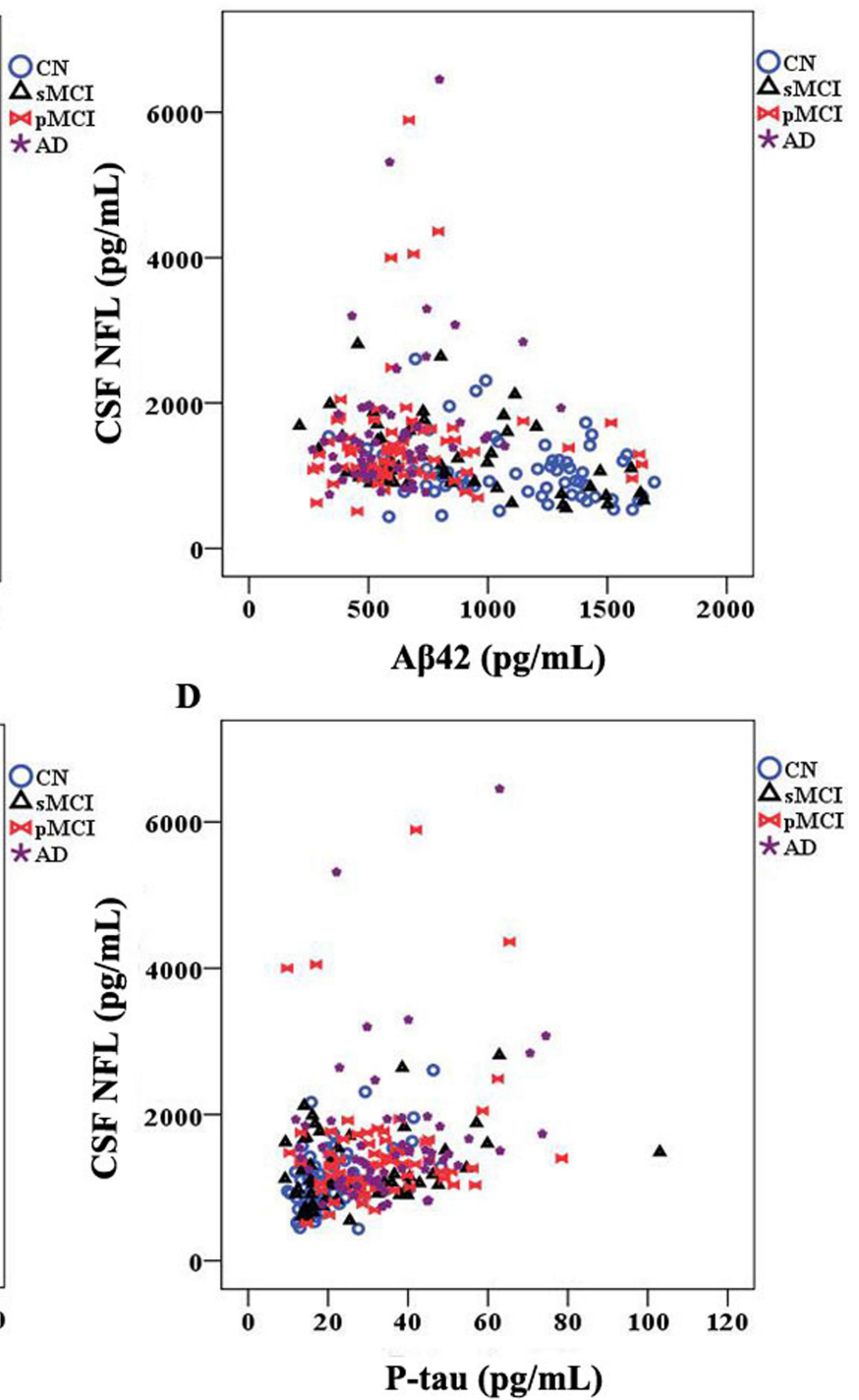

Fig. 2. CSF NFL in relation to plasma NFL and other biomarkers. Relationships between CSF NFL and plasma NFL(A) and A $\beta 42$ (B)and tau biomarkers (C and D) in different groups.
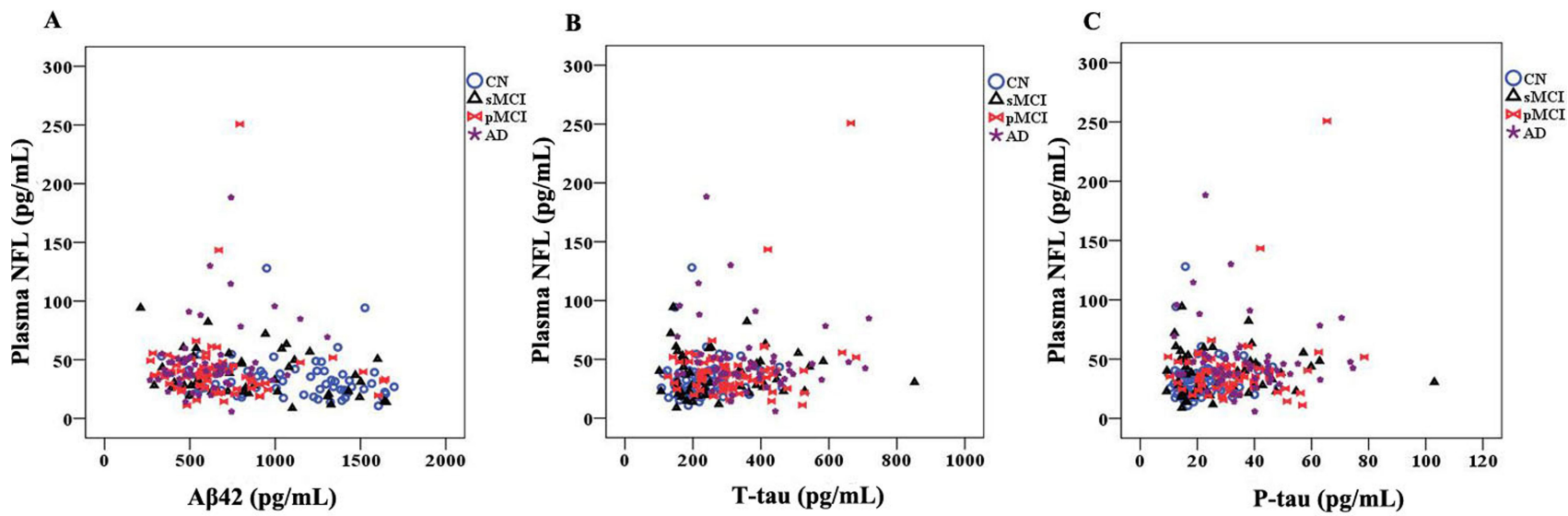

Fig. 3. Plasma NFL in relation to other biomarkers. There are relationships between plasma NFL and A $\beta 42(\mathrm{~A})$ and tau biomarkers (B and C) in different groups. 
Table 2. Correlations between NFL and other biomarkers.

\begin{tabular}{lcccc}
\hline & CN & sMCI & pMCI & AD \\
\hline CSF NFL vs Plasma NFL & $r=0.514(p<0.001)$ & $r=0.603(p<0.001)$ & $r=0.530(p<0.001)$ & $r=0.371(p=0.005)$ \\
CSF NFL vs CSF A $\beta 42$ & $r=-0.245(p=0.046)$ & $r=0.001(p=0.996)$ & $r=0.195(p=0.109)$ & $r=0.237(p=0.076)$ \\
CSF NFL vs CSF T-tau & $r=0.514(p<0.001)$ & $r=0.094(p=0.502)$ & $r=0.049(p=0.687)$ & $r=0.284(p=0.032)$ \\
CSF NFL vs CSF P-tau & $r=0.472(p<0.001)$ & $r=0.052(p=0.711)$ & $r=-0.031(p=0.799)$ & $r=0.284(p=0.063)$ \\
Plasma NFL vs CSF A $\beta 42$ & $r=-0.125(p=0.313)$ & $r=-0.044(p=0.752)$ & $r=0.030(p=0.805)$ & $r=0.247(p=0.065)$ \\
Plasma NFL vs CSF T-tau & $r=0.076(p=0.543)$ & $r=-0.020(p=0.885)$ & $r=0.285(p=0.018)$ & $r=-0.132(p=0.327)$ \\
Plasma NFL vs CSF P-tau & $r=0.042(p=0.737)$ & $r=-0.047(p=0.738)$ & $r=0.202(p=0.097)$ & $r=-0.142(p=0.292)$ \\
\hline
\end{tabular}

Table 3. AUC of biomarkers.

\begin{tabular}{lccccc}
\hline & CSF NFL & Plasma NFL & CSF and Plasma NFL & T-tau & P-tau \\
\hline CN vs sMCI & $0.604(0.501-0.706)$ & $0.575(0.470-0.681)$ & $0.613(0.510-0.715)$ & $0.578(0.469-0.687)$ & $0.592(0.484-0.699)$ \\
& $(p=0.053)$ & $(p=0.159)$ & $(p=0.036)$ & $(p=0.146)$ & $(p=0.087)$ \\
CN vs pMCI & $0.719(0.632-0.805)$ & $0.630(0.536-0.724)$ & $0.716(0.629-0.803)$ & $0.775(0.695-0.854)$ & $0.781(0.701-0.860)$ \\
& $(p<0.001)$ & $(p=0.009)$ & $(p<0.001)$ & $(p<0.001)$ & $(p<0.001)$ \\
CN vs AD & $0.760(0.676-0.844)$ & $0.728(0.638-0.818)$ & $0.781(0.702-0.861)$ & $0.811(0.733-0.889)$ & $0.811(0.732-0.891)$ \\
& $(p<0.001)$ & $(p<0.001)$ & $(p<0.001)$ & $(p<0.001)$ & $(p<0.001)$ \\
\hline
\end{tabular}
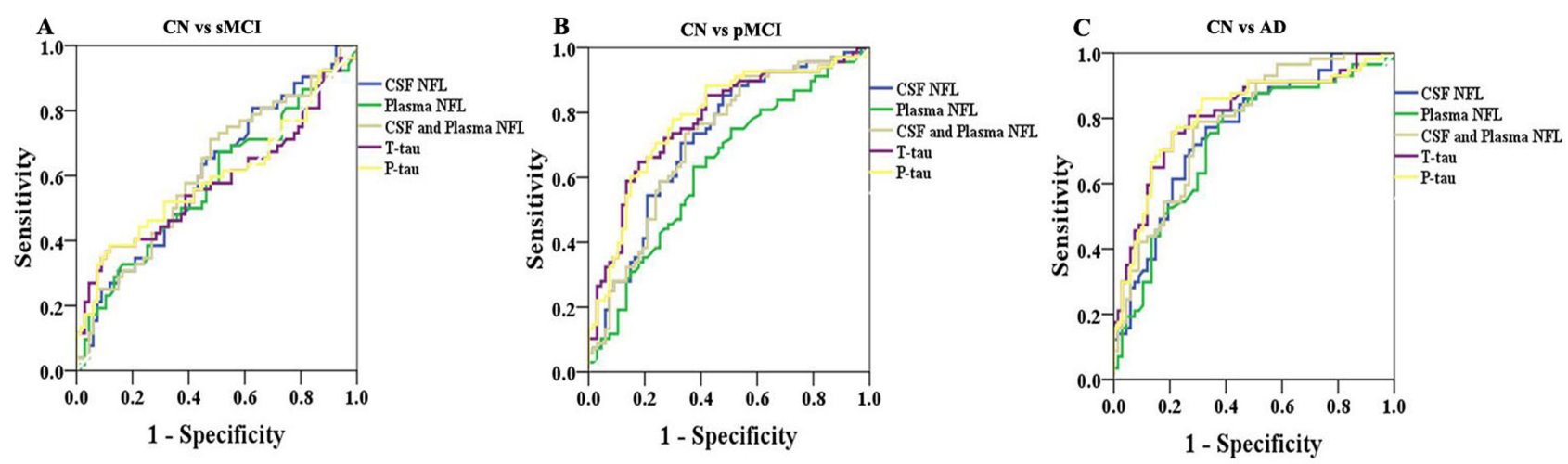

Fig. 4. ROC analyses. ROC analyses were used to assess the CSF biomarkers associated with clinical diagnoses for sMCI (A), pMCI (B), and AD (C).

and $\mathrm{AD}$ (Fig. 4C and Table 3). CSF T-tau and P-tau, CSF NFL and plasma NFL had almost the same diagnostic accuracy in pMCI and AD (Fig. 4B,C and Table 3).

\subsection{CSF NFL predicts conversion from MCI to $A D$}

$17 \mathrm{CN}$ participants progressed to $\mathrm{MCI}$ or $\mathrm{AD}$ dementia during the follow-up period, and $68 \mathrm{MCI}$ patients developed $\mathrm{AD}$ dementia. We tested whether baseline concentrations of CSF NFL and plasma NFL predicted conversion from CN to $\mathrm{MCI}$ or $\mathrm{AD}$ dementia and from $\mathrm{MCI}$ to $\mathrm{AD}$ dementia. CSF NFL and plasma NFL were used as continuous variables to establish Cox proportional hazard models. CSF NFL did not significantly predict conversion from $\mathrm{CN}$ to $\mathrm{MCI}$ or AD dementia (Fig. 5A). However, individuals with high CSF NFL, corresponding to MCI individuals whose CSF NFL were $\geq 1533 \mathrm{pg} / \mathrm{mL}$, progressed much more rapidly to dementia than subjects with lower levels $(<1533 \mathrm{pg} / \mathrm{mL}$, corresponding to the lower median levels of CSF NFL) $(p=0.049)$ (Fig. 5B). Moreover, plasma NFL could not predict the conversion from $\mathrm{CN}$ to $\mathrm{MCI}$ or $\mathrm{AD}$ dementia (Fig. 5C) and $\mathrm{MCI}$ to $\mathrm{AD}$ dementia (Fig. 5D).

\subsection{NFL in relation to baseline cognition}

High CSF NFL related to low MMSE $(\beta=-0.267, p=$ $0.023)$ (Fig. 6A) and high ADAS- $\operatorname{cog} 11(\beta=0.325, p=0.043)$ (Fig. 6B) at baseline in $\mathrm{AD}$, and with high ADAS-cog 11 in $\operatorname{sMCI}(\beta=0.285, p=0.045)$ (Fig. 6B). High plasma NFL correlated with low MMSE at baseline in $\operatorname{AD}(\beta=-0.241, p=$ 0.011) (Fig. 6C), as well as with high ADAS-cog 11 in sMCI $(\beta=0.306, p=0.002)$ (Fig. 6D). However, there were not any relationships between NFL and cognition in CN and pMCI (Fig. 6A-D).

\subsection{NFL in relation to baseline metabolism and brain structure}

CSF NFL correlated with baseline FDG-PET in CN ( $\beta=$ $-0.442, p=0.006)$ and $\mathrm{AD}(\beta=-0.378, p=0.011)$, but not in sMCI and pMCI (Fig. 7A). There was a trend for relationship between CSF NFL and FDG-PET in pMCI, but this did not reach statistical significance $(\beta=-0.242, p=0.067$ ) (Fig. 7A). High CSF NFL was associated with hippocampal atrophy in $\mathrm{CN}(\beta=-0.361, p=0.042)$, $\operatorname{sMCI}(\beta=-0.118, p=0.029)$, and $\operatorname{pMCI}(\beta=-0.126, p=0.019)$, but not in AD (Fig. $7 \mathrm{~B})$. High CSF NFL was associated with larger ventricules in $\mathrm{CN}(\beta=$ 


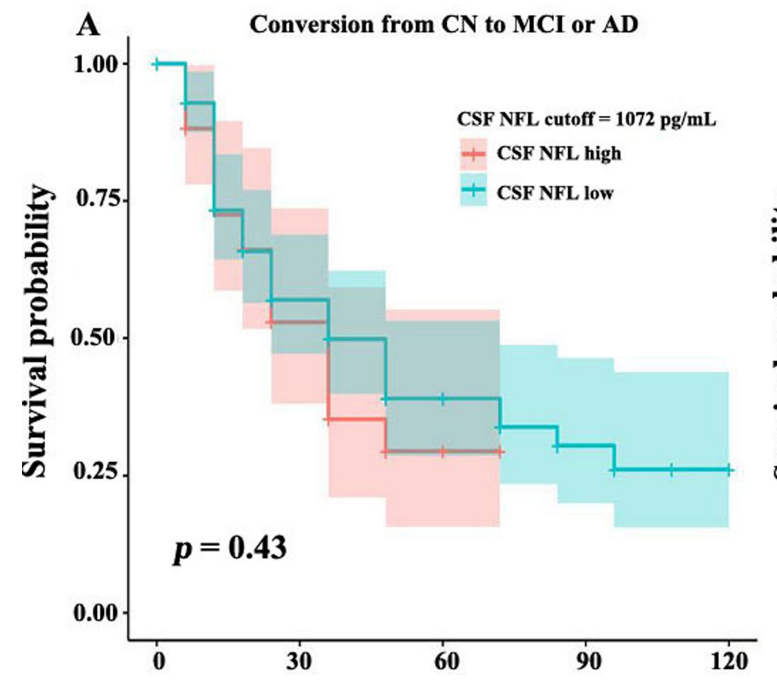

C

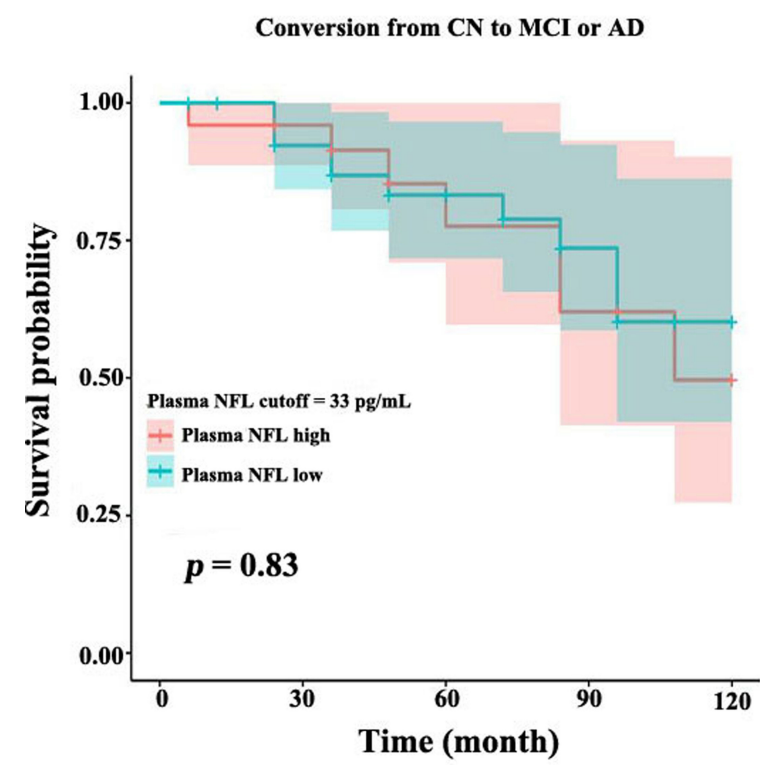

B

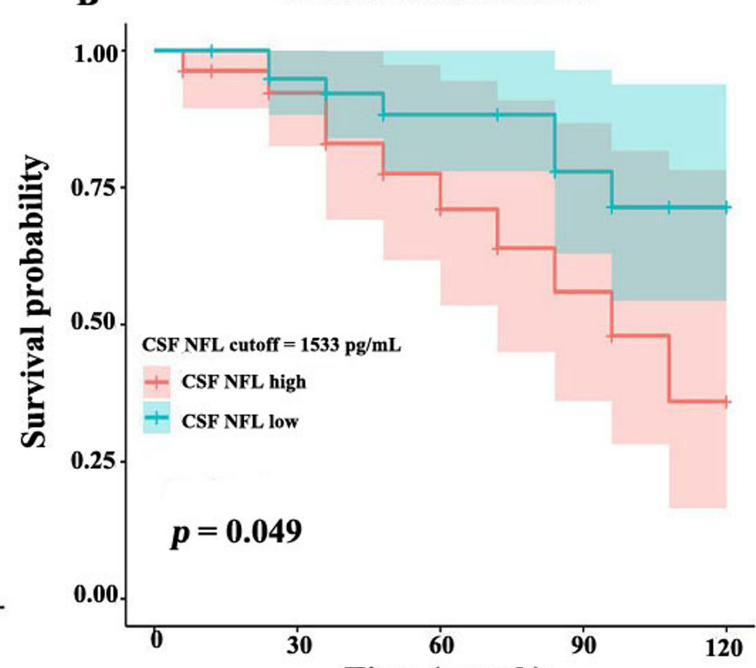

D

Time (month)

Conversion from MCI to AD

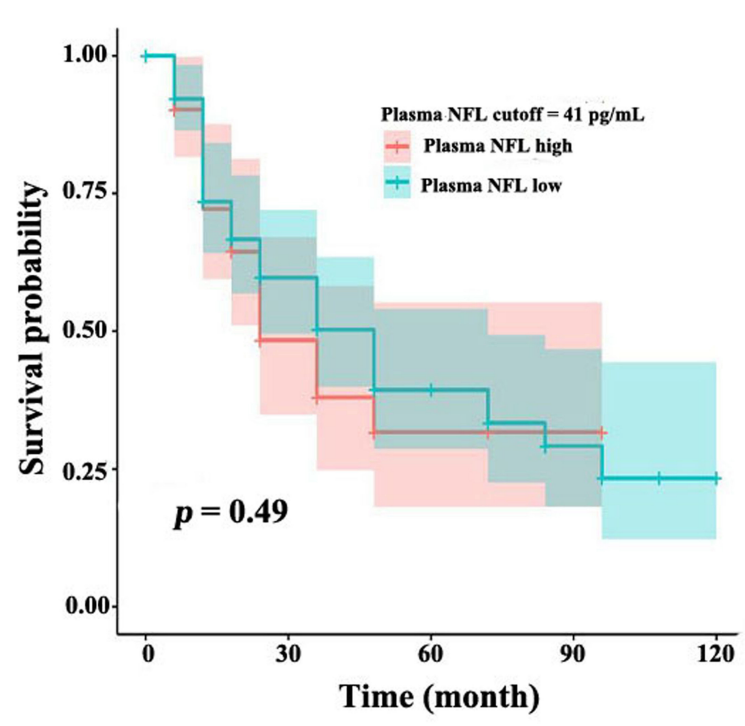

Fig. 5. Baseline CSF and plasma NFL as predictors of conversion from $\mathrm{CN}$ to $\mathrm{MCI}$ or $\mathrm{AD}$ and $\mathrm{MCI}$ to $\mathrm{AD}$. Conversion from $\mathrm{CN}$ to $\mathrm{MCI}$ or $\mathrm{AD}$ (A) and MCI to AD (B) as a function of CSF NFL. Conversion from CN to MCI or AD (C) and MCI to AD (D) as a function of plasma NFL.

$0.411, p=0.021)$, sMCI $(\beta=0.214, p=0.041)$, and $\operatorname{AD}(\beta=$ $0.327, p=0.014$ ), but not in pMCI (Fig. 7C).

Plasma NFL correlated with baseline FDG-PET in CN $(\beta$ $=-0.246, p=0.026)$ and $\operatorname{sMCI}(\beta=-0.252, p=0.021)$, but not in pMCI and AD (Fig. 7D). High plasma NFL associated with hippocampal atrophy in every diagnostic group $(\beta=-0.283$, $p=0.039$ for $\mathrm{CN} ; \beta=-0.312, p=0.032$ for sMCI; $\beta=-0.267$, $p=0.042$ for $\mathrm{pMCI} ; \beta=-0.279, p=0.044$ for AD) (Fig. $7 \mathrm{E}$ ). High plasma NFL was also related to larger ventriculuar volume in $\mathrm{CN}(\beta=0.268, p=0.040)$, $\operatorname{sMCI}(\beta=0.296, p=0.042)$, and $\operatorname{AD}(\beta=0.227, p=0.045)$, but not in pMCI (Fig. $7 \mathrm{~F})$.

\section{Discussion}

We evaluated the relationships between CSF and plasma NFL in MCI and AD patients from the ADNI database. We have the following major findings: firstly, CSF and plasma NFL concentrations were significantly elevated in AD. CSF NFL, but not plasma NFL, was significantly increased in pMCI relative to CN. Secondly, CSF and plasma NFL had almost the same diagnostic accuracy as CSF T-tau and P-tau for pMCI and AD. Our third main finding is that individuals with high CSF NFL progressed much more rapidly to $\mathrm{AD}$ than subjects with lower levels. Finally, CSF and plasma NFL are associated with baseline cognition in sMCI and AD and baseline cerebral metabolism and brain structure at some stages of $\mathrm{AD}$.

In line with a previous study [13], we report that CSF NFL was significantly higher in pMCI and AD dementia than $\mathrm{CN}$ individuals. We also observed there were significant differences between sMCI and AD. However, contrary to some ex- 

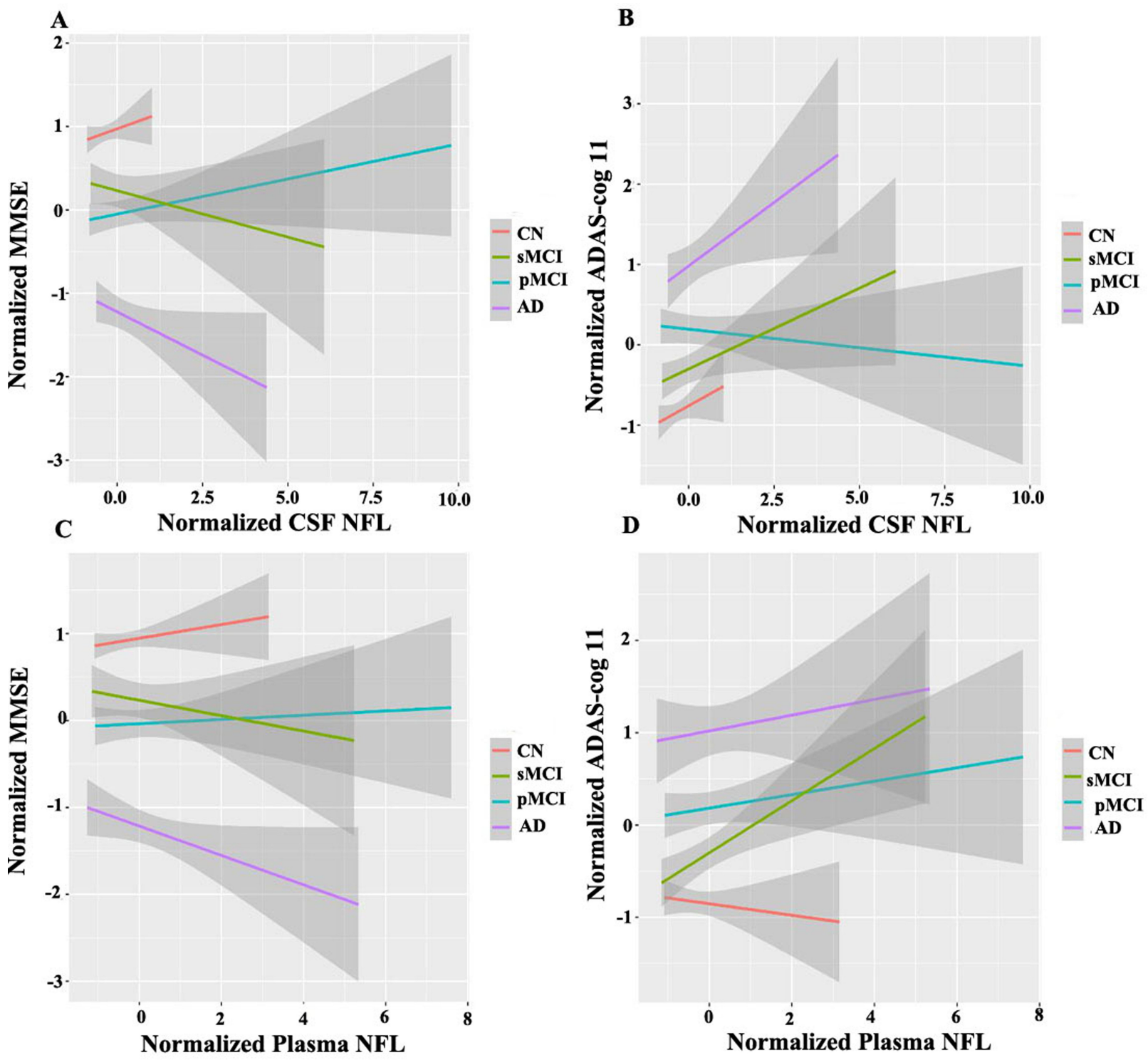

Fig. 6. CSF and plasma NFL in relation to baseline cognition. MMSE (A) and ADAS-Cog (B) at baseline function as baseline CSF NFL in different groups. MMSE (C) and ADAS-Cog (D) at baseline function as baseline plasma NFL in different groups. Values of all indicators are normalized.

isting reports $[14,15,17]$, plasma NFL in our study was significantly different between $\mathrm{CN}$ and $\mathrm{AD}$ and between sMCI and $\mathrm{AD}$. Still, there were no significant differences among $\mathrm{CN}$, sMCI, and pMCI. One explanation for these differences is that the number of cases in our study is relatively small, and the sampling bias is large. Moreover, in the present study, the correlations between CSF and plasma NFL with other CSF biomarkers were usually not statistically significant in the diagnostic groups. This finding may confirm the presence of several different pathological conditions in AD (such as $\mathrm{A} \beta$ pathological characteristics, tau pathological changes, different types of axonal degeneration) and drive different biomarker reactions, which are generally weakly correlated [14].

In our study, compared with CSF T-tau and P-tau, CSF and plasma NFL had almost the same diagnostic accuracy for
pMCI and AD. However, CSF and plasma NFL combined did not significantly improve the diagnostic accuracy for pMCI and $\mathrm{AD}$ over either biomarker in isolation. Interestingly, the combination of CSF and plasma NFL provided significantly different diagnostic accuracy for sMCI in our study, but CSF NFL, plasma NFL, CSF T-tau, and CSF P-tau had no similar effects, indicating that the combination of CSF and plasma NFL may be useful for the diagnosis of prodromal stages of $\mathrm{AD}$. In the future, we should verify its specificity for the diagnosis of sMCI in other neurodegenerative diseases.

There is increasing evidence that axonal degeneration is a surrogate indicator of $\mathrm{AD}$ neurodegeneration progression $[1,28]$. Although it has been shown that the combination of plasma NFL and MMSE could reliably predict whether MCI patients might progress to AD dementia within 5 years [29]. To our knowledge, no studies using Cox proportional haz- 

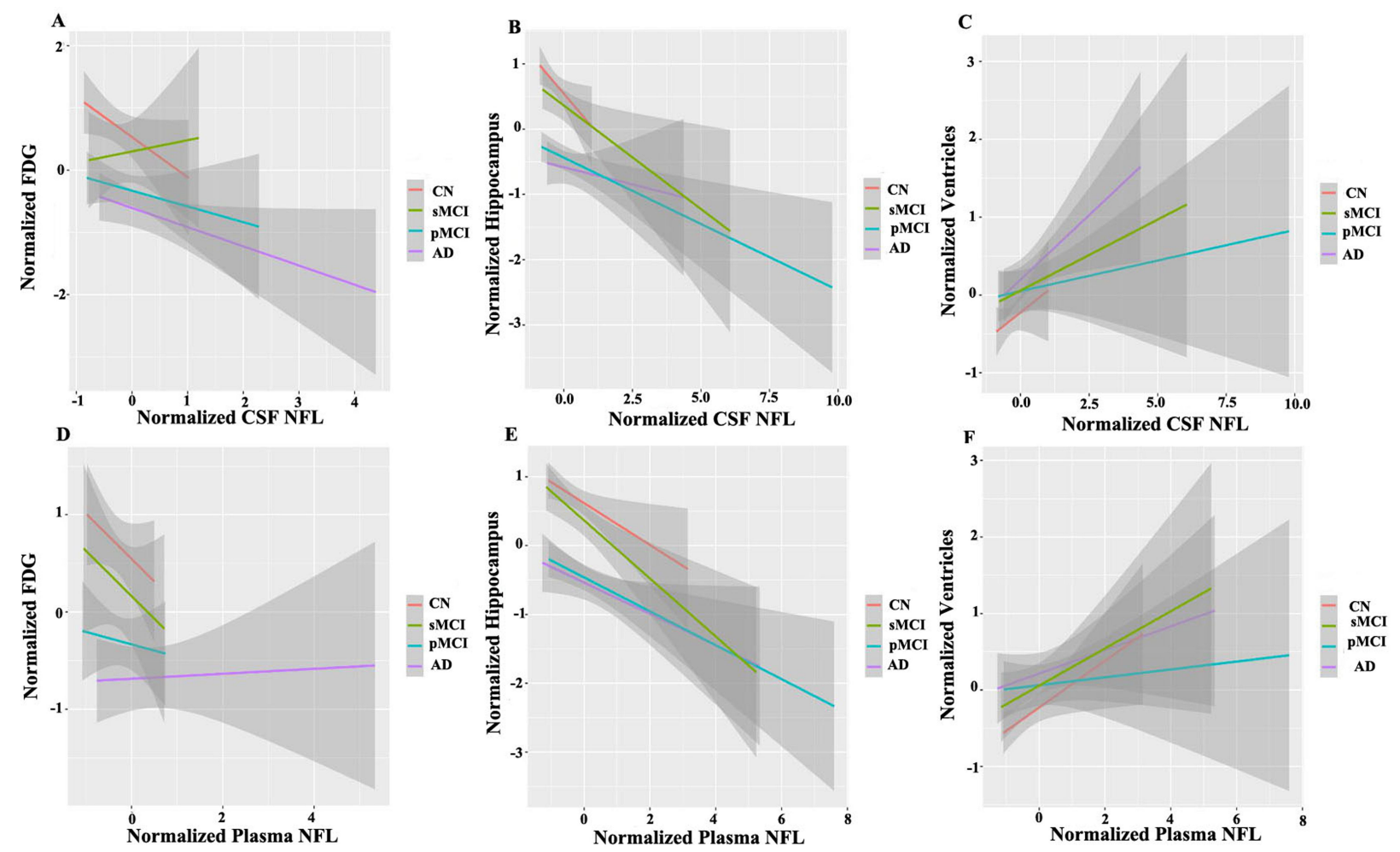

Fig. 7. CSF and plasma NFL in relation to baseline metabolism and brain structure. FDG (A), hippocampal volumes (B), and ventricular volumes (C) at baseline as a function of baseline CSF NFL in different groups. FDG (D), hippocampal volumes (E), and ventricular volumes (F) at baseline as a function of baseline plasma NFL in different groups. Values of all indicators are normalized.

ard models have evaluated the predictive value of CSF NFL for the conversion from $\mathrm{CN}$ to $\mathrm{MCI}$ or $\mathrm{AD}$ and from $\mathrm{MCI}$ to AD. Here, we demonstrate that CSF NFL but not plasma NFL provides predictive value for future disease progression in MCI participants. Our findings indicate that CSF NFL may contribute to the prognostic roles of CSF A $\beta 42$, T-tau, and $\mathrm{P}$-tau in predicting the evolution of cognitive impairment.

Previous research has reported that plasma NFL might be a promising biomarker of progressive cognitive decline in elderly patients with MCI [30]. We examined the associations between CSF or plasma NFL and cognition. Statistically significant interactions were found at baseline for the sMCI group and MMSE or ADAS-cog 11 and the AD group and MMSE or ADAS-cog 11. These results suggest that CSF and plasma NFL were more strongly associated with the cognitive outcomes in sMCI and AD than in $\mathrm{CN}$ at baseline. It has been shown that in the MCI group, higher CSF NFL concentrations were related to faster brain atrophy over time, as measured by changes in whole-brain volume, ventricular volume, and hippocampal volume [13]. In addition, Mattsson et al. [14] reported that elevated plasma NFL was associated with AD-related atrophy (baseline and overtime) and brain hypometabolism (over time).

Our results show that CSF and plasma NFL were correlated with several baseline imaging measures of $\mathrm{AD}$ related neurodegeneration, including low cortical glucose metabolism, hippocampal atrophy, and ventricular enlarge- ment. These relationships were particularly pronounced with CSF and plasma NFL in relation to large ventricles in $\mathrm{CN}$, sMCI, and AD groups. These results indicate that AD's cognition, brain structure, and cortical metabolism may be related to axonal degeneration. However, there were some inconsistencies in the correlation between NFL and cognition and between NFL and brain metabolism or structure. For example, the correlations between MMSE or ADAS-Cog and CSF or plasma NFL in pMCI patients were the opposite of all other groups.

Similarly, brain metabolism correlated well with plasma NFL in $\mathrm{CN}$ and sMCI but not in $\mathrm{pMCI}$ and $\mathrm{AD}$. Vice versa CSF NFL correlated well with baseline FDG brain metabolism in $\mathrm{CN}$ and $\mathrm{AD}$, but not in $\mathrm{PMCI}$ and sMCI, suggesting that they were confounded by diagnosis. We do not know the exact reason, but it indicates that cognition, brain metabolism, and brain structure may be related to many factors, and NFL is only one of them.

CSF and plasma NFL concentrations are significantly higher in other neurodegenerative diseases other than $\mathrm{AD}$ than healthy controls [28, 31-34]. In addition, although a previous study investigated that CSF NFL had the potential to help distinguish frontotemporal dementia from $\mathrm{AD}$ and Parkinson disease from atypical Parkinson's syndrome [34], the diagnostic usefulness of CSF and plasma NFL might be limited due to the finding that it is also elevated in other neurodegenerative diseases, indicating low specificity for AD. 


\section{Conclusions}

Our results support the use of NFL as a biomarker of axonal degeneration. CSF and plasma NFL were significantly elevated in AD. In addition, compared with CSF T-tau and P-tau, CSF and plasma NFL had similar diagnostic accuracy for pMCI and AD. Moreover, high CSF NFL predicted conversion from MCI to AD. Finally, high CSF and plasma NFL are related to poor cognition, low metabolism, hippocampal atrophy, and ventricular enlargement at some stages of AD. These findings may suggest the potential use of NFL in trial designs, monitoring response to therapies, treatment decisions, and outcome evaluations.

\section{Abbreviations}

$\mathrm{A} \beta$, amyloid- $\beta$; $\mathrm{AD}$, Alzheimer's disease; ADAS-cog, Alzheimer's disease assessment scale-cog; ADNI, Alzheimer's disease Neuroimaging Initiative; ANOVA, Analysis of covariance; $A P O E$, Apolipoprotein E; AUC, area under the curve; CDR, Clinical Dementia Rating scale; CN, cognitively normal; CSF, cerebrospinal fluid; FDG-PET, 18FFluorodeoxyglucose-PET; HR, hazard ratios; MCI, mild cognitive impairment; MMSE, Mini-mental State Examination; MRI, magnetic resonance imaging; NFL, neurofilament light; PET, positron emission tomography; ROC, receiver operating curve.

\section{Author contributions}

YC-manuscript drafting. JT-manuscript drafting. JLanalysis of data. MB-analysis and interpretation of data. HZ-critical review of manuscript and study supervision.

\section{Ethics approval and consent to participate}

The Institutional Review Boards of all participating institutions approved the ADNI study. All participants in each centre obtained informed written consent. ADNI makes all its data publicly available. Our manuscript was approved and accepted for submission by the ADNI Data and Publications Committee (ADNI DPC). For more details, please see: http://adni.loni.usc.edu/data-samples/access-data/.

\section{Acknowledgment}

Data collection and sharing for this project was funded by the Alzheimer's Disease Neuroimaging Initiative (ADNI) (National Institutes of Health Grant U01 AG024904) and DOD ADNI (Department of Defense award number W81XWH-12-2-0012). ADNI is funded by the National Institute on Aging, the National Institute of Biomedical Imaging and Bioengineering, and through generous contributions from the following: AbbVie, Alzheimer's Association; Alzheimer's Drug Discovery Foundation; Araclon Biotech; BioClinica, Inc.; Biogen; Bristol-Myers Squibb Company; CereSpir, Inc.; Cogstate; Eisai Inc.; Elan Pharmaceuticals, Inc.; Eli Lilly and Company; EuroImmun; F. Hoffmann-La Roche Ltd and its affiliated company Genentech, Inc.; Fujirebio; GE Healthcare; IXICO Ltd.;
Janssen Alzheimer Immunotherapy Research \& Development, LLC.; Johnson \& Johnson Pharmaceutical Research \& Development LLC.; Lumosity; Lundbeck; Merck \& Co., Inc.; Meso Scale Diagnostics, LLC.; NeuroRx Research; Neurotrack Technologies; Novartis Pharmaceuticals Corporation; Pfizer Inc.; Piramal Imaging; Servier; Takeda Pharmaceutical Company; and Transition Therapeutics. The Canadian Institutes of Health Research is providing funds to support ADNI clinical sites in Canada. Private sector contributions are facilitated by the Foundation for the National Institutes of Health (www.fnih.org). The grantee organization is the Northern California Institute for Research and Education, and the study is coordinated by the Alzheimer's Therapeutic Research Institute at the University of Southern California. ADNI data are disseminated by the Laboratory for Neuro Imaging at the University of Southern California.

\section{Funding}

This study was supported by the Medical Research Project of Chongqing Healthy Committee (2018MSXM058).

\section{Conflict of interest}

The authors declare no conflict of interest.

\section{References}

[1] Blennow K, de Leon MJ, Zetterberg H. Alzheimer's disease. Lancet. 2006; 368: 387-403.

[2] Alzheimer's Association. 2016 Alzheimer's Disease Facts and Figures. Alzheimer's Dement. 2016; 12: 459-509.

[3] Egan MF, Kost J, Voss T, Mukai Y, Aisen PS, Cummings JL, et al. Randomized Trial of Verubecestat for Prodromal Alzheimer's Disease. New England Journal of Medicine. 2019; 380: 1408-1420.

[4] Knopman DS. Lowering of Amyloid-Beta by $\beta$-Secretase Inhibitors-some Informative Failures. New England Journal of Medicine. 2019; 380: 1476-1478.

[5] Selkoe DJ. Alzheimer disease and aducanumab: adjusting our approach. Nature Reviews Neurology. 2019; 15: 365-366.

[6] Honig LS, Vellas B, Woodward M, Boada M, Bullock R, Borrie M, et al. Trial of Solanezumab for Mild Dementia Due to Alzheimer's Disease. New England Journal of Medicine. 2018; 378: 321-330.

[7] Jack CR, Bennett DA, Blennow K, Carrillo MC, Dunn B, Haeberlein SB, et al. NIA-AA Research Framework: toward a biological definition of Alzheimer's disease. Alzheimer's \& Dementia. 2019; 14: 535-562.

[8] Yu J, Tan L, Hardy J. Apolipoprotein E in Alzheimer's disease: an update. Annual Review of Neuroscience. 2015; 37: 79-100.

[9] Gozes I. Specific protein biomarker patterns for Alzheimer's disease: improved diagnostics in progress. EPMA Journal. 2019; 8 : 255-259.

[10] Fuchs E, Cleveland DW. A structural scaffolding of intermediate filaments in health and disease. Science. 1998; 279: 514-519.

[11] Norgren N, Rosengren L, Stigbrand T. Elevated neurofilament levels in neurological diseases. Brain Research. 2003; 987: 25-31.

[12] Rosengren LE, Karlsson JE, Karlsson JO, Persson LI, Wikkelsø C. Patients with amyotrophic lateral sclerosis and other neurodegenerative diseases have increased levels of neurofilament protein in CSF. Journal of Neurochemistry. 1996; 67: 2013-2018.

[13] Zetterberg H, Skillbäck T, Mattsson N, Trojanowski JQ, Portelius E, Shaw LM, et al. Association of Cerebrospinal Fluid Neurofilament Light Concentration with Alzheimer Disease Progression. JAMA Neurology. 2016; 73: 60-67.

[14] Mattsson N, Andreasson U, Zetterberg H, Blennow K. Association 
of Plasma Neurofilament Light with Neurodegeneration in Patients with Alzheimer Disease. JAMA Neurology. 2017; 74: 557566.

[15] Mattsson N, Cullen NC, Andreasson U, Zetterberg H, Blennow K. Association between Longitudinal Plasma Neurofilament Light and Neurodegeneration in Patients with Alzheimer Disease. JAMA Neurology. 2019; 76: 791-799.

[16] Gaiottino J, Norgren N, Dobson R, Topping J, Nissim A, Malaspina A, et al. Increased neurofilament light chain blood levels in neurodegenerative neurological diseases. PLoS ONE. 2014; 8: e75091.

[17] Zhou W, Zhang J, Ye F, Xu G, Su H, Su Y, et al. Plasma neurofilament light chain levels in Alzheimer's disease. Neuroscience Letters. 2018; 650: 60-64.

[18] Weiner MW, Veitch DP, Aisen PS, Beckett LA, Cairns NJ, Cedarbaum J, et al. 2014 Update of the Alzheimer's Disease Neuroimaging Initiative: a review of papers published since its inception. Alzheimer's \& Dementia. 2015; 11: 1-120.

[19] Berg L. Clinical Dementia Rating (CDR). Psychopharmacology Bulletin. 1988; 24: 637-639.

[20] Folstein MF, Folstein SE, McHugh PR. "Mini-mental state". A Practical Method for Grading the Cognitive State of Patients for the Clinician. Journal of Psychiatric Research. 1975; 12: 189-198.

[21] Aisen PS, Petersen RC, Donohue MC, Gamst A, Raman R, Thomas RG, et al. Clinical Core of the Alzheimer's Disease Neuroimaging Initiative: progress and plans. Alzheimer's \& Dementia. 2010; 6: 239-246.

[22] Tierney MC, Fisher RH, Lewis AJ, Zorzitto ML, Snow WG, Reid DW, et al. The NINCDS-ADRDA Work Group criteria for the clinical diagnosis of probable Alzheimer's disease: a clinicopathologic study of 57 cases. Neurology. 1988; 38: 359-364.

[23] Portelius E, Zetterberg H, Skillbäck T, Törnqvist U, Andreasson $\mathrm{U}$, Trojanowski JQ, et al. Cerebrospinal fluid neurogranin: relation to cognition and neurodegeneration in Alzheimer's disease. Brain. 2016; 138: 3373-3385.

[24] Shaw LM, Vanderstichele H, Knapik-Czajka M, Clark CM, Aisen PS, Petersen RC, et al. Cerebrospinal fluid biomarker signature in Alzheimer's disease neuroimaging initiative subjects. Annals of Neurology. 2009; 65: 403-413.

[25] Mattsson N, Insel PS, Palmqvist S, Portelius E, Zetterberg H, Weiner $\mathrm{M}$, et al. Cerebrospinal fluid tau, neurogranin, and neurofilament light in Alzheimer's disease. EMBO Molecular Medicine. 2017; 8: 1184-1196.
[26] Risacher SL, Saykin AJ. Neuroimaging and other biomarkers for Alzheimer's disease: the changing landscape of early detection. Annual Review of Clinical Psychology. 2013; 9: 621-648.

[27] Landau SM, Mintun MA, Joshi AD, Koeppe RA, Petersen RC, Aisen PS, et al. Amyloid deposition, hypometabolism, and longitudinal cognitive decline. Annals of Neurology. 2013; 72: 578-586.

[28] Skillbäck T, Farahmand B, Bartlett JW, Rosén C, Mattsson N, Nägga K, et al. CSF neurofilament light differs in neurodegenerative diseases and predicts severity and survival. Neurology. 2015; 83: 1945-1953.

[29] Darmanthé N, Tabatabaei-Jafari H, Cherbuin N. Combination of Plasma Neurofilament Light Chain and Mini-Mental State Examination Score Predicts Progression from Mild Cognitive Impairment to Alzheimer's Disease within 5 Years. Journal of Alzheimer's Disease. 2021; 82: 951-964.

[30] He L, Morley JE, Aggarwal G, Nguyen AD, Vellas B, de Souto Barreto P. Plasma Neurofilament Light Chain Is Associated With Cognitive Decline in Non-dementia Older Adults. Scientific Reports. 2021; 11:13394.

[31] Kovacs GG, Andreasson U, Liman V, Regelsberger G, Lutz MI, Danics $\mathrm{K}$, et al. Plasma and cerebrospinal fluid tau and neurofilament concentrations in rapidly progressive neurological syndromes: a neuropathology-based cohort. European Journal of Neurology. 2017; 24: 1326-1377.

[32] Delaby C, Alcolea D, Carmona-Iragui M, Illán-Gala I, MorenasRodríguez E, Barroeta I, et al. Differential levels of Neurofilament Light protein in cerebrospinal fluid in patients with a wide range of neurodegenerative disorders. Scientific Reports. 2020; 10: 9161.

[33] Olsson B, Portelius E, Cullen NC, Sandelius, Zetterberg H, Andreasson $\mathrm{U}$, et al. Association of Cerebrospinal Fluid Neurofilament Light Protein Levels with Cognition in Patients with Dementia, Motor Neuron Disease, and Movement Disorders. JAMA Neurology. 2019; 76: 318-325.

[34] Bridel C, van Wieringen WN, Zetterberg H, Tijms BM, Teunissen CE, Alvarez-Cermeño JC, et al. Diagnostic Value of Cerebrospinal Fluid Neurofilament Light Protein in Neurology. JAMA Neurology. 2019; 76: 1035-1048. 